\title{
Quantification of the Effect of Circumferential Repeated 3D Features on Radial Displacement of a Compressor Casing Focusing Model Simplification: Part I
}

\author{
Tobias Schmidt ${ }^{1}$, Volker Gümmer ${ }^{1}$, Marco Konle ${ }^{2}$ \\ ${ }^{1}$ Technische Universität München - Institute for Turbomachinery and Flight Propulsion \\ 85748 Garching, Germany \\ Tobias.Schmidt@ltf.mw.tum.de; Volker.Guemmer@1tf.mw.tum.de \\ ${ }^{2}$ MTU Aero Engines AG \\ 80995 München, Germany \\ Marco.Konle@mtu.de
}

\begin{abstract}
Within the framework of the development of a preliminary aero engine compressor casing design methodology, prompt tip clearance assessments between casing and rotor blades are aspired. Based on the Finite Element Method and proceeding from the 3D model of a generic axial compressor casing, the intention is to first investigate and quantify the effect of various 3D features on radial displacement during steady state engine operation. Bleed air ports and bolted joints are the 3D features this work is devoted to address. Parameter studies varying the circumferential quantity and dimension of the stated 3D features are conducted for purposeful validity. Constructive on these deliverables and the desire for reduced preprocessing and computational time consumption, the transition from 3D to 2D axisymmetric Finite Element modeling by implementing diverse model simplifications is focused. Considering the numerical effort towards prompt clearance assessments, reducing the nodal quantity of the computational grid through transition from $3 \mathrm{D}$ to $2 \mathrm{D}$ is an expedient issue that has to be resumed in future work, since it turned out that the circumferential and longitudinal track generated by the 3D features covered can be approximately neglected.
\end{abstract}

Keywords: Compressor, Radial Casing Displacement, Finite Element Analysis, 3D Features, Model Simplification.

\section{Introduction}

Considering a reduced time to market from the first concept towards the final product implementation, the preliminary design stage of multidisciplinary driven subjects like aero engine development involves essential potential for process optimization. This work reports the initial results from an industry-sponsored project dedicated to setting up a rapid preliminary and target-oriented design methodology for future compressor casing structures. Proceeding from an initial geometry and covering various approaches for numerical model simplification, the intended long-term goal relates to prompt tip clearance assessments between compressor casing and rotor. Regarding approaches to numerical model simplification, the application of metamodeling techniques like surrogate modeling in particular is emphasized as one of the project targets.

At first, the effects of circumferential repeated 3D features on radial displacement of a generic compressor casing have to be addressed and quantified. Compressor casings of modern aero engines involve many different 3D features. Bolted joints, bleed air ports, different types of stator stage and compressor casing designs are just a few of the features that will be investigated via Finite Element Analysis and methods for model simplifications will be elaborated.

In aero engine axial compressor design, high system efficiency as well as operational stability are constrained by clearance-induced losses. The tendency of development towards lower blade heights exacerbates these losses due to the increased relative ratio between blade height and tip clearance. Thus, within future aero engine development reductions in tip clearance are of elevated significance for further upgrades even in the thrust-to-weight ratio and service life. Another challenge that has to be encountered during transient engine operation is to keep the tip clearance at a minimum, but large enough to prevent a collision between the blade tip and the casing. The scope of this elaboration is restricted to symmetrical variations in tip clearance. Hence, the expansion behavior of the casing model variants investigated in this work is a consequence of thermal as well as pressure driven forces. 


\section{Methodology}

For quantification of the effect of 3D features on radial displacement of a compressor casing, coupled thermomechanical implicit simulations are conducted via the commercial Finite Element software Abaqus FEA. The 3D features focused in this work are restricted to bolted joints (BJ) and bleed air ports (BAP). Based on the red framed casing construction in the general arrangement presented in Figure 1, a single-walled high-pressure compressor casing geometry has been generated in 3D $\mathrm{CAD}$ first. The section selected for 3D feature analysis consists of two casing rings, one stator row, and respectively one arrangement for bleed air ports and bolted joints (see Figure 2). Referring to the red dashed section in Figure 1 and with respect to subsequent correlation analysis when extending a given compressor casing to a multistage variant, a standardized part design for additional casing rings and stators was chosen.

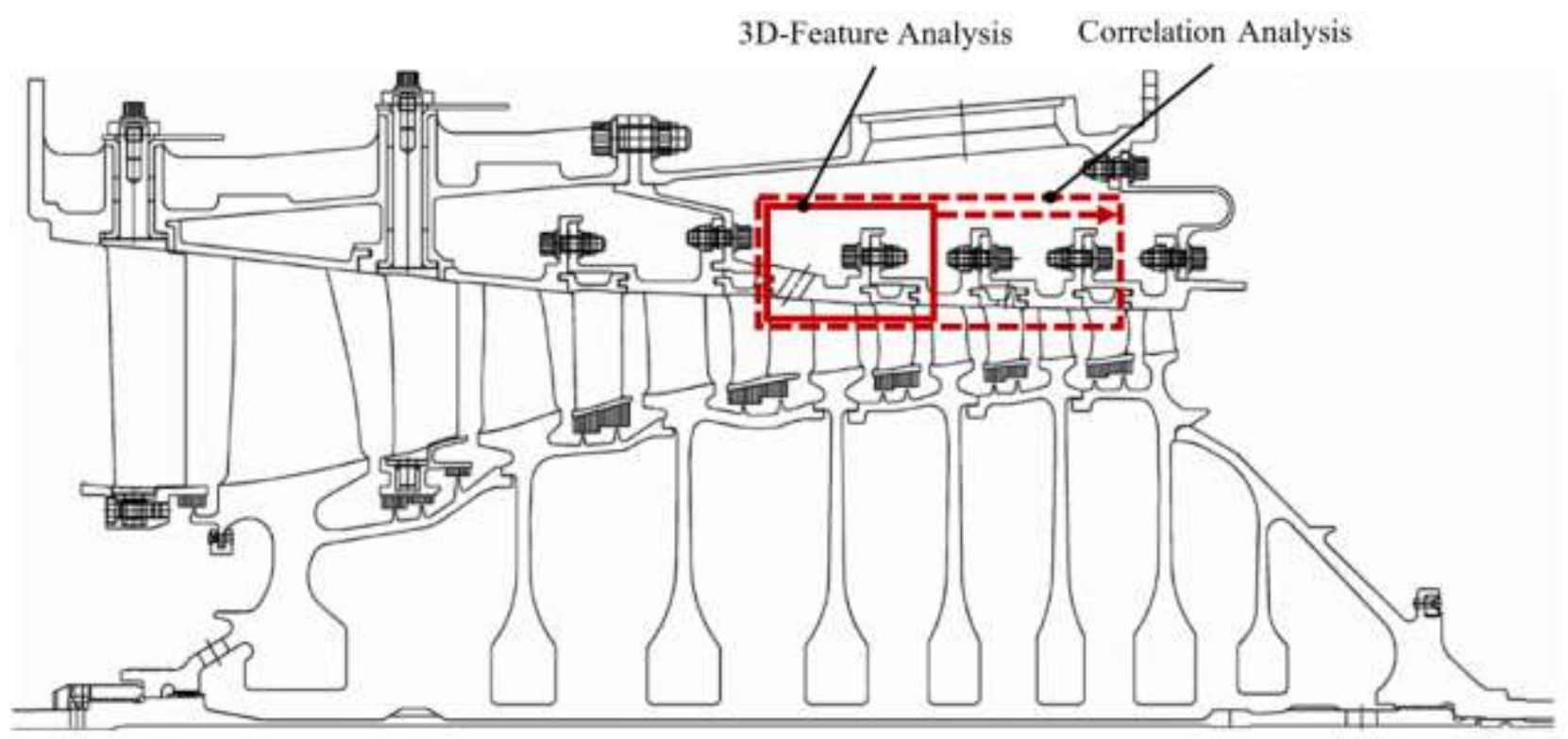

Fig. 1: Generic Compressor General Arrangement and Modeled Sections [1 mod.].

Circling back to identify the effect of bleed air ports and bolted joints on radial displacement, the circumferential quantity (CQ) and diameter size $(\varnothing)$ of these 3D-Features were varied. Considering the rotational axis for bleed air ports as well as bolted joints and dependent on circumferential repetition, all model variants share the same $z-r$-plane positioning. Table 1 lists the resulting model variants involved in the parameter studies conducted and compared as proposed in Table 2 . Since a correlation was assessed between the effects of bleed air ports and bolted joints on radial displacement, separate sensitivity analysis was performed supplementary for each of both 3D features.

Table 1: Model Setup Variants Considered for Parameter Studies.

\begin{tabular}{|c|c|c|c|c|}
\hline Model Setup & BAP CQ & BJ CQ & BAP $\emptyset$ & BJ $\varnothing$ \\
\hline Variant I & 36 & 0 & $7 \mathrm{~mm}$ & $0 \mathrm{~mm}$ \\
\hline Variant II & 36 & 36 & $7 \mathrm{~mm}$ & $8 \mathrm{~mm}$ \\
\hline Variant III & 36 & 72 & $7 \mathrm{~mm}$ & $8 \mathrm{~mm}$ \\
\hline Variant IV & 0 & 36 & $0 \mathrm{~mm}$ & $8 \mathrm{~mm}$ \\
\hline Variant V & 72 & 36 & $7 \mathrm{~mm}$ & $8 \mathrm{~mm}$ \\
\hline Variant VI & 36 & 36 & $7 \mathrm{~mm}$ & $7 \mathrm{~mm}$ \\
\hline Variant VII & 36 & 36 & $7 \mathrm{~mm}$ & $9 \mathrm{~mm}$ \\
\hline Variant VIII & 36 & 36 & $10 \mathrm{~mm}$ & $8 \mathrm{~mm}$ \\
\hline
\end{tabular}


Table 2: Flow Chart of Model Comparison and the thereby Focused Objectives.

\begin{tabular}{|c|c|c|c|}
\hline \multicolumn{3}{|c|}{ Model Comparison } & Objective \\
\hline Variant I & Variant II & Variant III & Impact of BJ CQ on Radial Displacement \\
\hline Variant IV & Variant II & Variant V & Impact of BAP CQ on Radial Displacement \\
\hline Variant II & Variant VI & Variant VII & Impact of BJ $\varnothing$ on Radial Displacement \\
\hline Variant IV & Variant II & Variant VIII & Impact of BAP $\varnothing$ on Radial Displacement \\
\hline
\end{tabular}

The geometry conceived for 3D feature analysis is demonstrated in Figure 2, which uses Variant II by way of example. As just mentioned, it consists of two casing rings, one stator row, and one respective arrangement for bleed air ports and bolted joints, while the latter 3D feature accounts for axial fixation. As pictured in the sectional view A-A, the $r$ - $z$-plane positioning of the rotational axis for bleed air ports and bolted joints remains unmodified for all model variants.
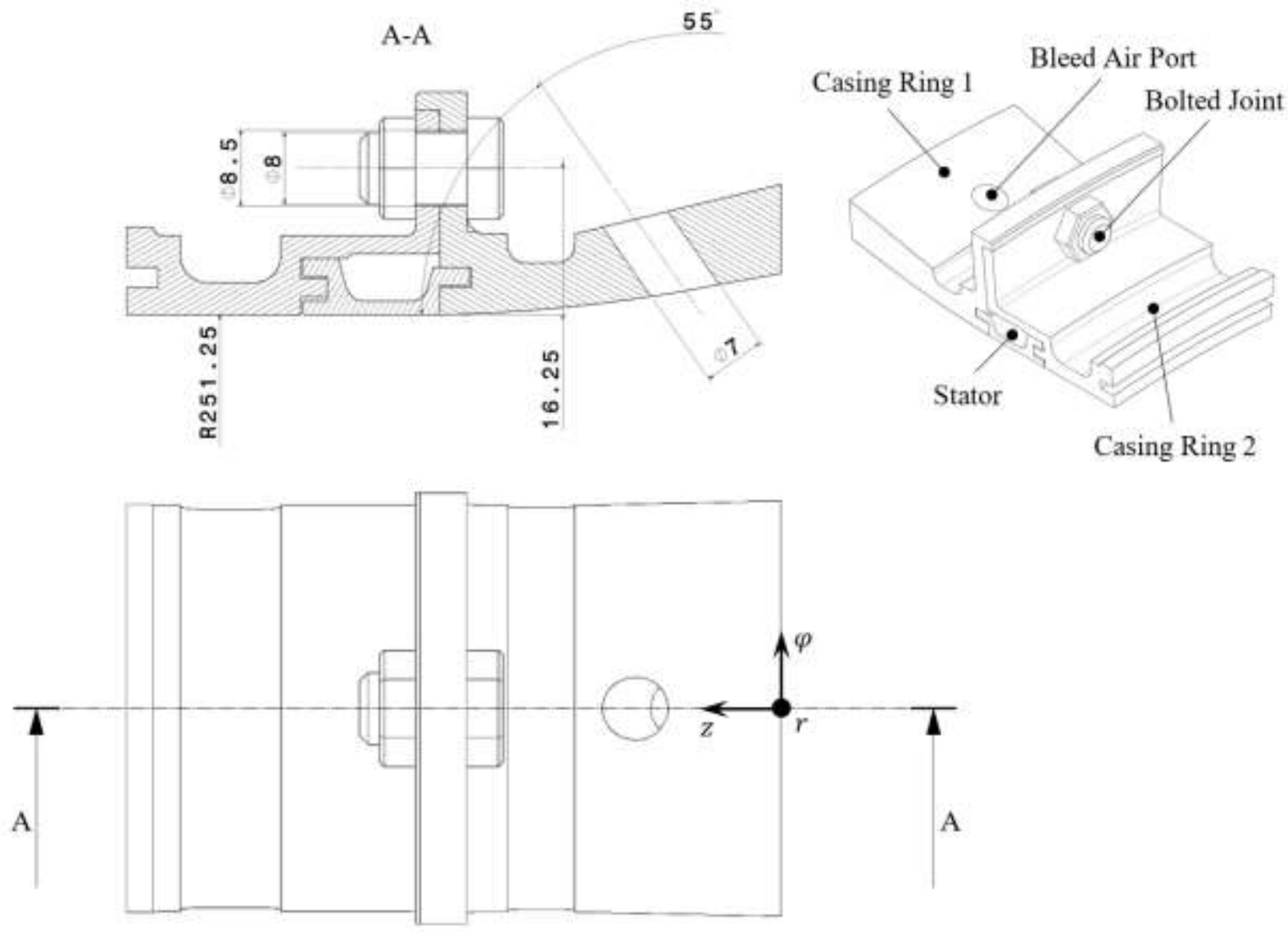

Fig. 2: Geometry and Sectional View A-A of Variant II.

The thermo-mechanical boundary conditions implemented are displayed in Figure 3. On the left side, index $a$ describes the surface of the casing wetted with bleed air, index $i$ the inner side of the casing exposed to the core flow, and index $B A P$ the bleed air port surfaces. $T$ symbolizes the temperature, $p$ the pressure, and $\alpha$ the heat transfer coefficient. The set of parameter values inserted can be extracted from Table 3. Due to rotational symmetry, only a sector of $10^{\circ}$ is modeled, and the cyclic symmetry condition is installed at the sector boundaries. Regarding part contact definition, tie constraints are used for heat transfer analysis and switched to surface-to-surface contact interactions to perform thermo-mechanical analysis with allowed part separation. Regarding fixation, the $r-\varphi$-plane surface at $z=0 \mathrm{~mm}$ was chosen to lock the degree of freedom 
in $z$-direction; the degree of freedom in $\varphi$-direction was fixed at the upper edge of this surface. Unless specified otherwise, the model setup is assigned to the cylindrical coordinate system.

Concerning Table 3, the initial condition constitutes the case for an unloaded structure. Subsequently increasing the gauge pressure and the temperature level represents the load resulting from the design point of a modern high-pressure compressor. In order to be as realistic as possible, the assigned core flow pressure $p_{i}$ and temperature $T_{i}$ are defined as $z$ dependent functions and increase in flow direction. In case of bleed air port existence an additional cylindrical coordinate system was created to model the pressure drop from $p_{i}$ to $p_{a}$, which depends on the bleed air port length $r_{B A P}$ characterized in Figure 3.

Table 3: Parameter Settings Imprinted on the Surfaces of the Generated Single-Flange Casing Structures.

\begin{tabular}{|c|c|c|c|}
\hline Location & Parameter & Initial Condition & Initial Load \\
\hline \multirow{3}{*}{ Hotgas Annulus } & $p_{i}$ & $0 \mathrm{MPa}$ & $(0.014 \cdot z+3) M P a$ \\
\hline & $T_{i}$ & $293 K$ & $1.097 \cdot z+740 K$ \\
\hline & $\alpha_{i}$ & {$[-]$} & $1000 \mathrm{~W} / \mathrm{m}^{2} \mathrm{~K}$ \\
\hline \multirow{3}{*}{ Bleed Air Chamber } & $p_{a}$ & $0 M P a$ & $3 M P a$ \\
\hline & $T_{a}$ & $293 K$ & $740 K$ \\
\hline & $\alpha_{a}$ & {$[-]$} & $50 \mathrm{~W} / \mathrm{m}^{2} \mathrm{~K}$ \\
\hline \multirow{3}{*}{ Bleed Air Port } & $p_{B A P}$ & $0 M P a$ & $\left(-0.0164 \cdot r_{B A P}+3.15\right) M P a$ \\
\hline & $T_{B A P}$ & $293 K$ & $740 \mathrm{~K}$ \\
\hline & $\alpha_{B A P}$ & {$[-]$} & $500 \mathrm{~W} / \mathrm{m}^{2} \mathrm{~K}$ \\
\hline
\end{tabular}
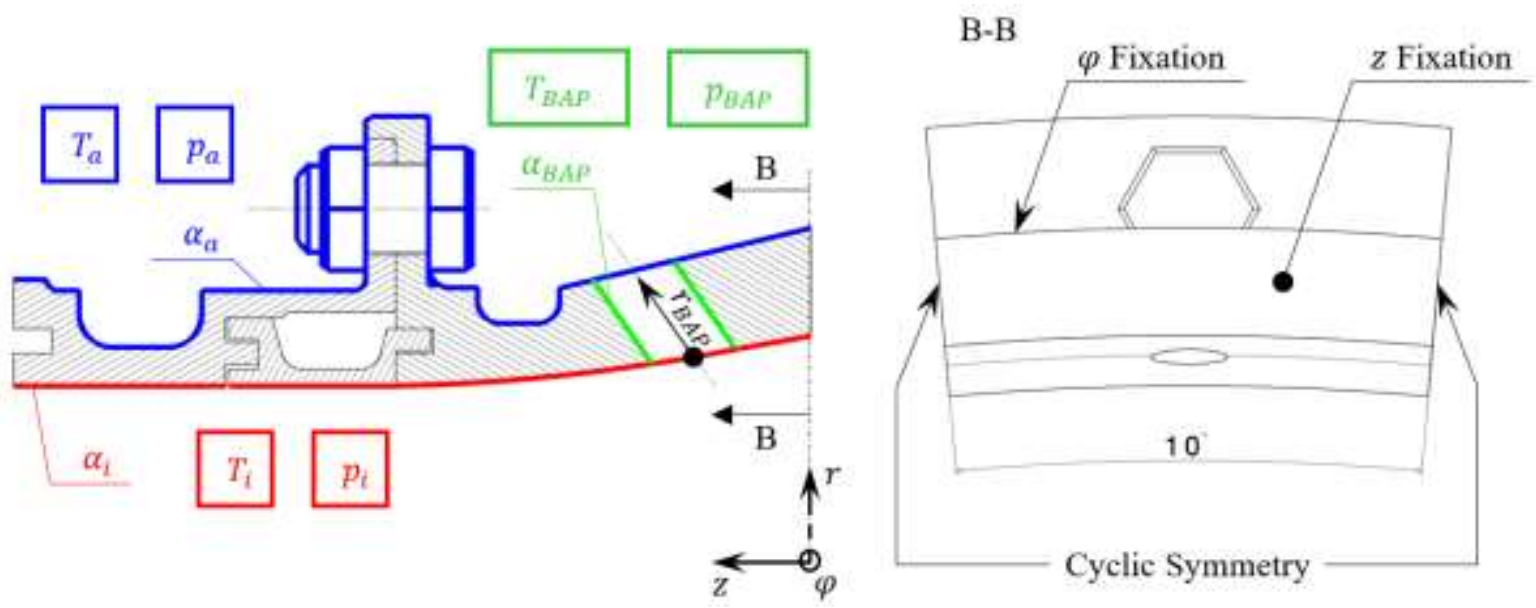

Fig. 3: Schematic of the Boundary and Load Conditions Implemented for the Single-Flange Casing Structures Investigated.

Due to fundamental studies concerning the effect of the 3D features focused in this work, Inconel 718 was selected as material for the casing constructions. The isotropic material model implemented contains temperature-dependent data for Young's modulus, Poisson's ratio, density, specific heat capacity, thermal expansion coefficient, and thermal heat conductance sourced from $[2,3,4]$. An extension to a casing assembly with a multi-material definition will be processed at a progressed stage of revision level. 
A hybrid computational grid consisting of approximately 150,000 second-order elements was created for each of the calculated casing constructions registered in Table 1 . The mesh resolution is good enough to deliver smooth and converged temperature and displacement result distributions. Attention was paid during partitioning to each contact surface in order to ensure even node-to-node contact between the part instances involved. Figure 4 presents the mesh of Variant III.

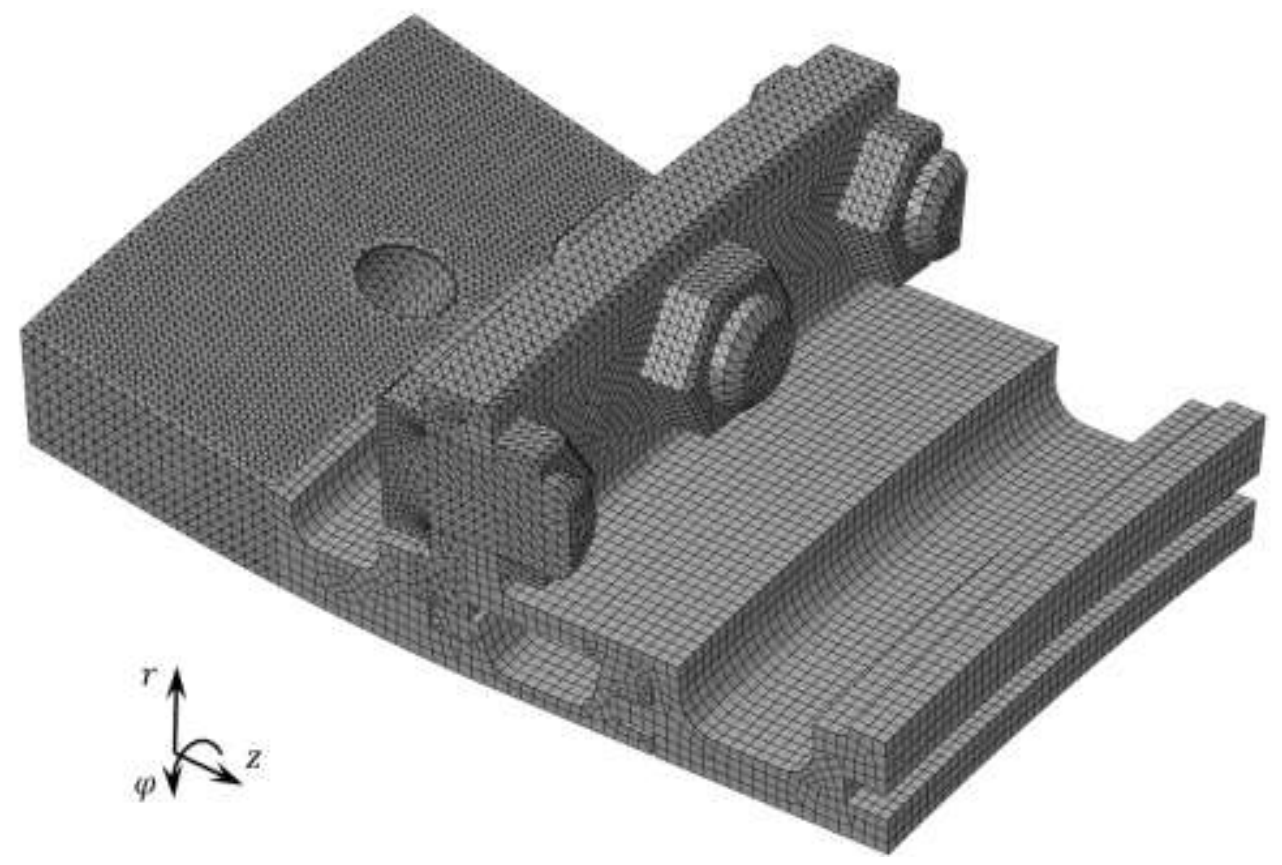

Fig. 4: Generated Mesh of Variant III.

The three paths shown in Figures 5 and 6 (indicated in red) were taken into account regarding evaluation of the Finite Element simulation results with a focus on radial casing displacement. Given that the inner casing surface in particular is relevant for future tip clearance assessments, all paths are located on this surface. The circumferential path A is devoted to analyzing the effects of bolted joints. The circumferential path B turned out to be well-suited for studying the effect of bleed air ports, and path $\mathrm{C}$ is intended for correlation analysis when extending a given compressor casing to a multistage variant. In addition, path $\mathrm{C}$ is involved for studying the effects of applied model simplifications when transferring from 3D to 2D axisymmetric Finite Element modeling.

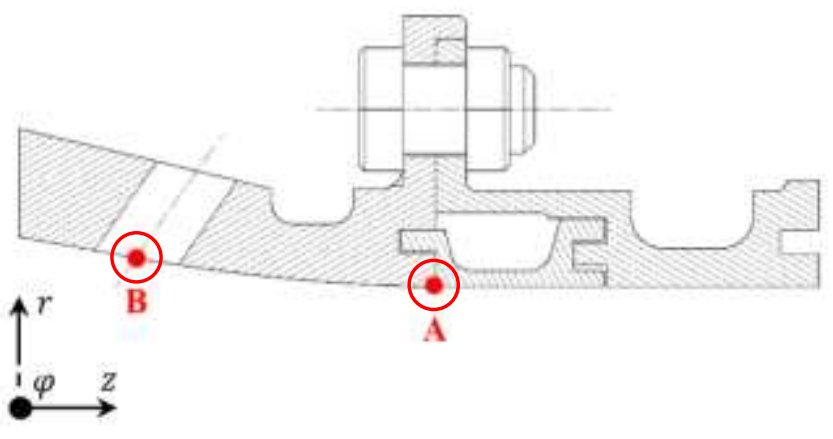

Fig. 5: Circumferential Paths A and B for 3D Feature Evaluation.

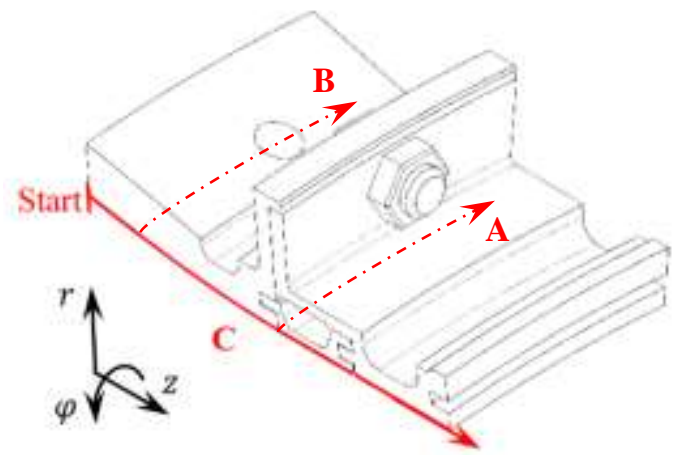

Fig. 6: Path C for Correlation Analysis and Circumferential Paths A and B. 


\section{Results and Discussion}

The Finite Element results achieved at initial load condition are presented and discussed in this section. In the following charts, the vertical and dashed black labels denote the normalized distance where the maximum deviation between each compared set of model variants was detected and calculated. From Figure 7 to Figure 10, the left figures show the results of model variants registered in Table 1, while the right figures visualize the separate 3D feature effect just covered. The model comparison was executed as proposed in Table 2.

\subsection{Effect of Bolted Joint $C Q$ and $\varnothing$ on Radial Casing Displacement}

Beginning with the impact of bolted joint CQ on radial casing displacement, Figure 7 presents the calculated values of Variants I, II and III along path A. The corresponding comparison between Variants II, VI and VII along path A for quantification of bolted joint $\varnothing$ on radial casing displacement is reproduced in Figure 8. Bolted joints generally induce a local peak in radial displacement.

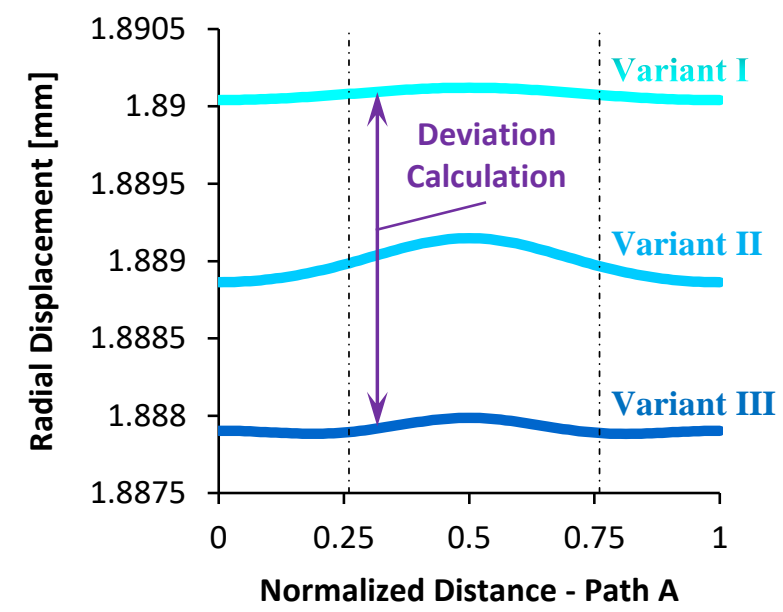

$B J C Q=0$

$B J C Q=36$

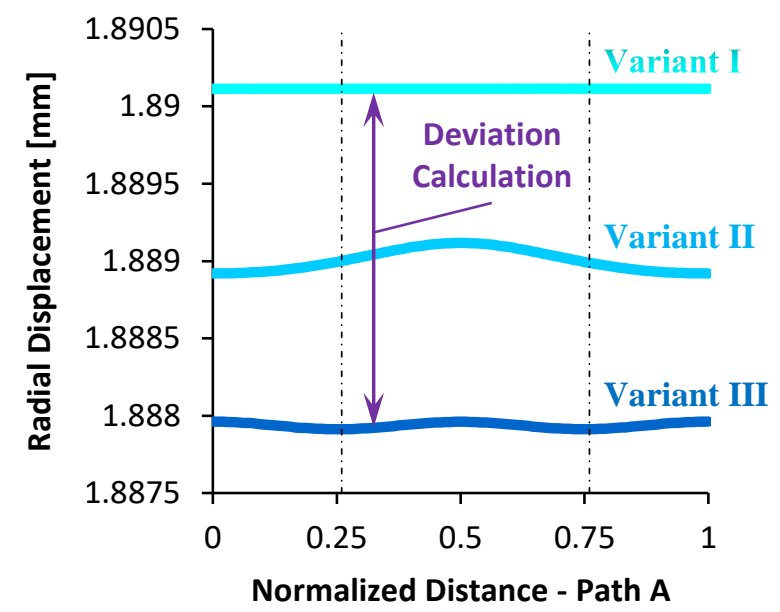

$B J C Q=72$
Maximum Deviation at -....- Labels

Fig. 7: Effect of Circumferential Quantity of Bolted Joints on Radial Displacement with (Left) and without (Right) One Prior Centrally Arranged Bleed Air Port.

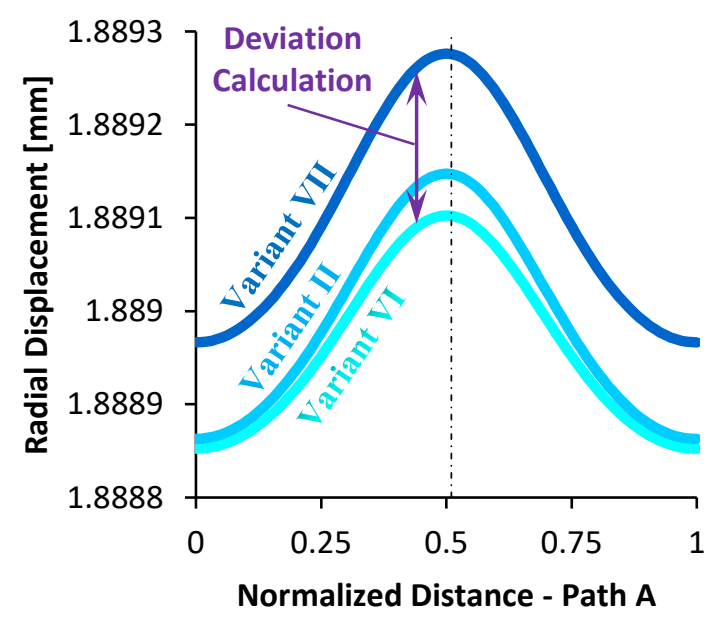

$B J \emptyset=7 \mathrm{~mm}$
$B J \emptyset=8 \mathrm{~mm}$

Fig. 8: Effect of Bolted Joint $\emptyset$ on Radial Displacement with (Left) and without (Right) One Prior Centrally Arranged Bleed Air Port.

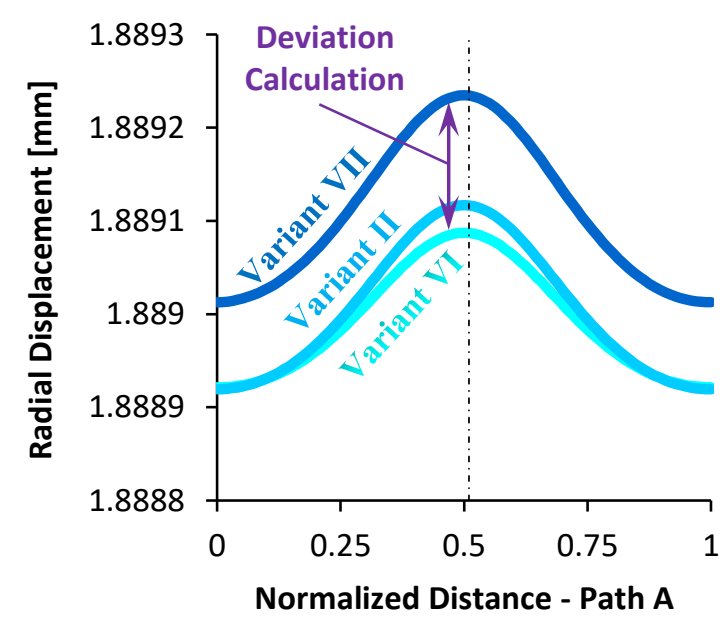

Maximum Deviation at -....- Labels 
Evaluating Figure 7, an increased CQ of bolted joints leads to an approximately proportional reduction of radial displacement. Due to an increased surface wetted with bleed air the dominant reason for this reaction is ascribed to a cooling effect. The maximum deviation between the compared models amounts to $0.116 \%$ in relation to Variant III and is localized at the normalized distances 0.26 and 0.76 along path A.

The opposite tendency but in a nonlinear way is recognized in Figure 8, where a growth of bolted joint $\emptyset$ causes risen radial displacements. The maximum deviation identified is covered by Variant VI and VII and localized along path A at normalized distance 0.51 . The calculated maximum deviation value in relation to Variant VI is less than $0.01 \%$.

\subsection{Effect of Bleed Air Port CQ and $\varnothing$ on Radial Casing Displacement}

The consequence of bleed air port CQ on radial casing displacement is visualized in Figure 9, comparing the calculated values of Variants IV, II and V along path B. For quantification of the effect of bleed air port $\varnothing$ on radial casing displacement, the results of Variants IV, II and VIII along path B are compared in Figure 10. Bleed air ports generally induce a local minimum in radial displacement.
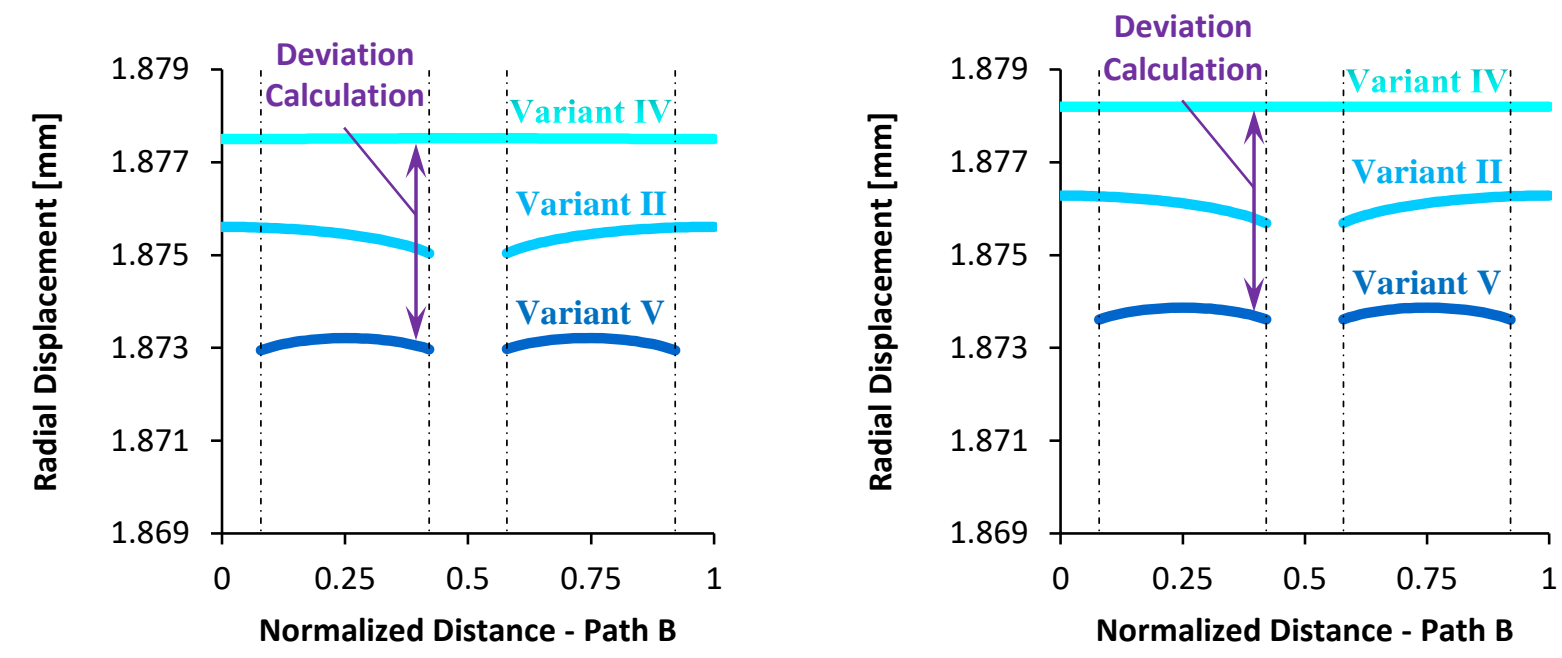
$B A P C Q=0$
$B A P C Q=36$
$B A P C Q=72$
Maximum Deviation
at -...- - Labels

Fig. 9: Effect of Circumferential Quantity of Bleed Air Ports on Radial Displacement with (Left) and without (Right) One Subsequent Centrally Arranged Bolted Joint.

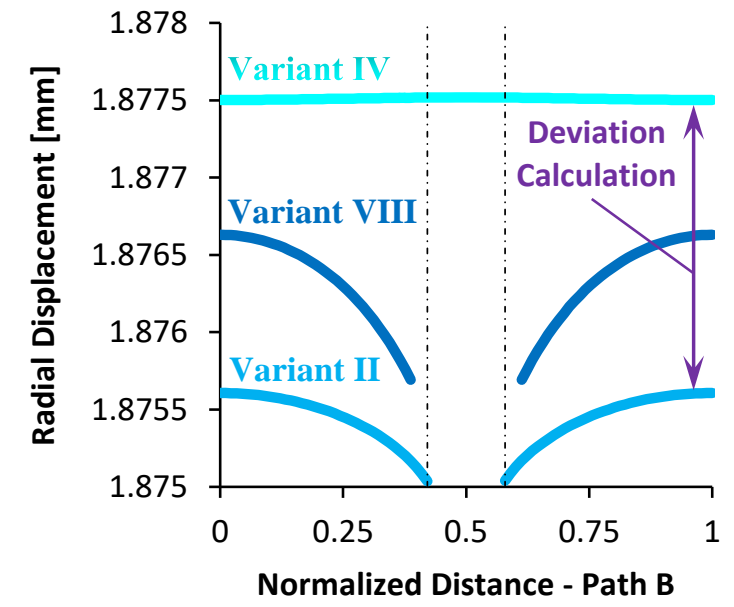

$B A P \emptyset=0 \mathrm{~mm}$ $B A P \emptyset=7 \mathrm{~mm}$

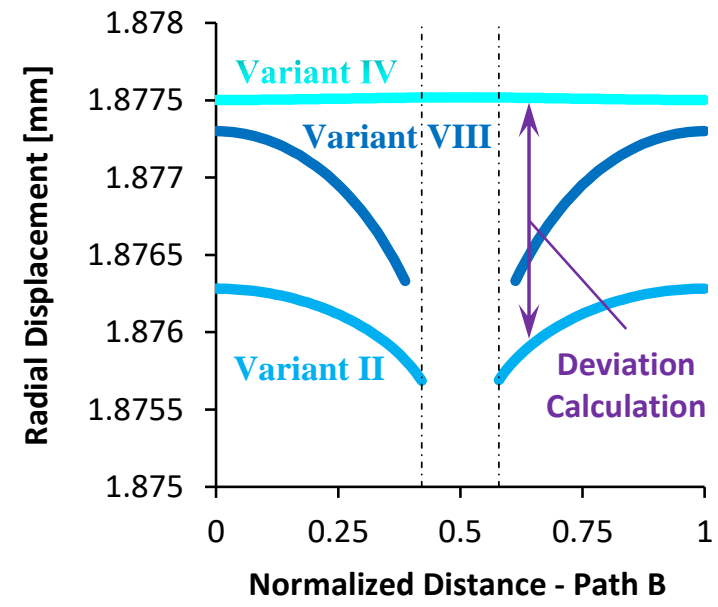

Maximum Deviation at -....- Labels

Fig. 10: Effect of Bleed Air Port $\emptyset$ on Radial Displacement with (Left) and without (Right) One Subsequent Centrally Arranged Bolted Joint. 
According to the results visualized in Figure 9, an increased CQ of bleed air ports causes an approximately proportional reduction of radial casing displacement. Again, the dominant reason for this reaction is attributed to a cooling effect. Consulting Variant IV and V, the maximum deviation in dimension of $0.245 \%$ is calculated in relation to Variant V and detected at normalized distances $0.079,0.421,0.579$ and 0.921 along path B.

On the contrary, Figure 10 reveals that, once there is existence of bleed air ports in casing geometry, a growth of bleed air port $\varnothing$ induces a rise in radial displacements. Involving Variant II and IV, the maximum deviation along path B is identified at normalized distances 0.421 and 0.579 and amounts to $0.134 \%$ in relation to Variant II.

\subsection{Effect of Model Extension on Radial Casing Displacement}

When extending the previous single-flange compressor casing model to a multistage variant with standardized part design, a global stiffening effect on radial displacement with increasing impact in $z$-direction is induced by the additional components. Along path C, Figure 11 demonstrates the tendency for Variant II as a single-flange model (in red) and as a two-flange model (in black). Both models share the equivalent boundary conditions specified in Table 3 . To better visualize the correlation, the blue curve shows the development of displacement deviation calculated according to equation (1) as an expression of stiffening.

$$
\text { Deviation }=\frac{\text { Radial Displacement }{ }_{2 \text { Flange Model }}-\text { Radial Displacement }_{1 \text { Flange Model }}}{\text { Radial Displacement }{ }_{2 \text { Flange Model }}}
$$

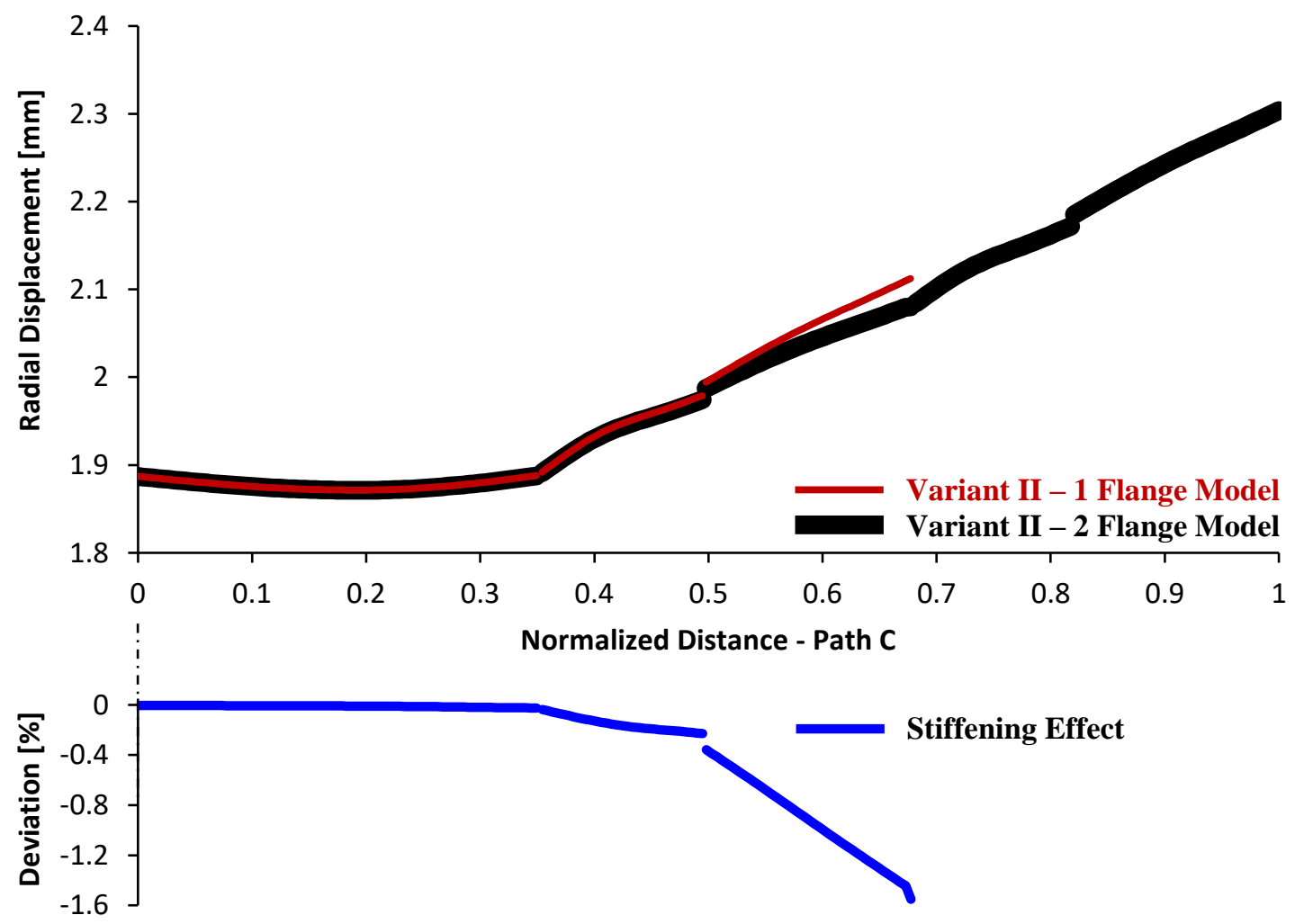

Fig. 11: Effect of Model Extension on Radial Casing Displacement.

\subsection{Effect of Model Simplification on Radial Casing Displacement}

The intention of this work revolves around investigating and quantifying the effect of circumferential repeated 3D features on radial casing displacement. Previous calculated dimensions of deviation between the compared model variants along path $\mathrm{A}$ and $\mathrm{B}$ lead to the insight that the effect on radial displacement caused by bolted joints and bleed air ports is approximately insignificant. Towards prompt tip clearance assessments between compressor casing and rotor the application of model simplifications is essential. Thus, the transition from $3 \mathrm{D}$ to $2 \mathrm{D}$ axisymmetric Finite Element modeling is 
investigated in the present section. Figure 12a compares the calculated radial displacement of Variant III (Detailed 3D Model) with stepwise applied model simplifications. First, bolted joints and bleed air ports are left aside (Simplified 3D Model). Subsequently, the transition from 3D to 2D was transacted by analyzing only the axisymmetric cross section of the simplified 3D model (2D Model). For quantification, Figure 12b illustrates the implicated effect on radial displacement deviation along path $\mathrm{C}$ when transitioning from detailed 3D Finite Element modeling towards 2D axisymmetric Finite Element modeling. Consequently, all of the 3D model variants listed in Table 1 are compared with the 2D axisymmetric model according to equation 2. Additionally, positions for future clearance assessments are indicated in Figure 12 with dashed lines.

a)

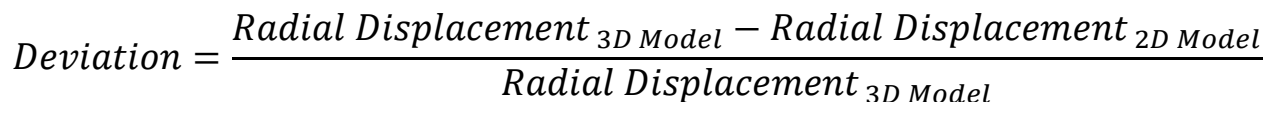

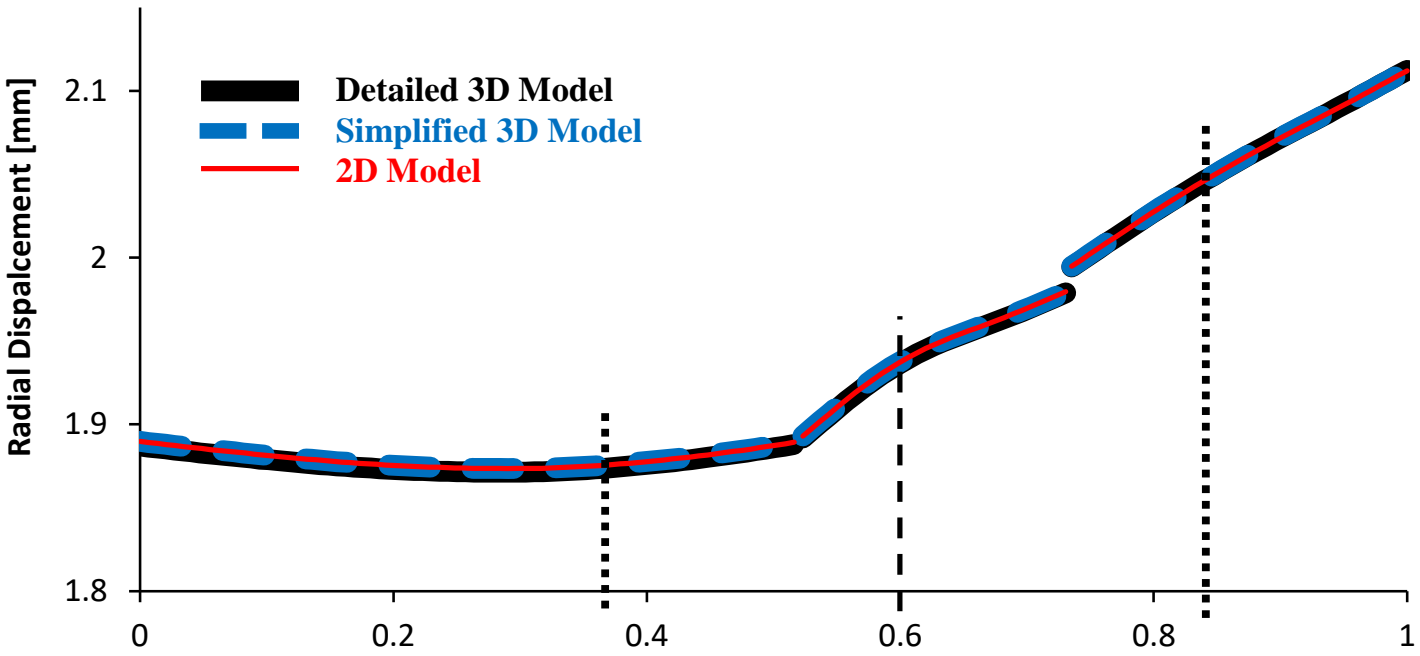

b)
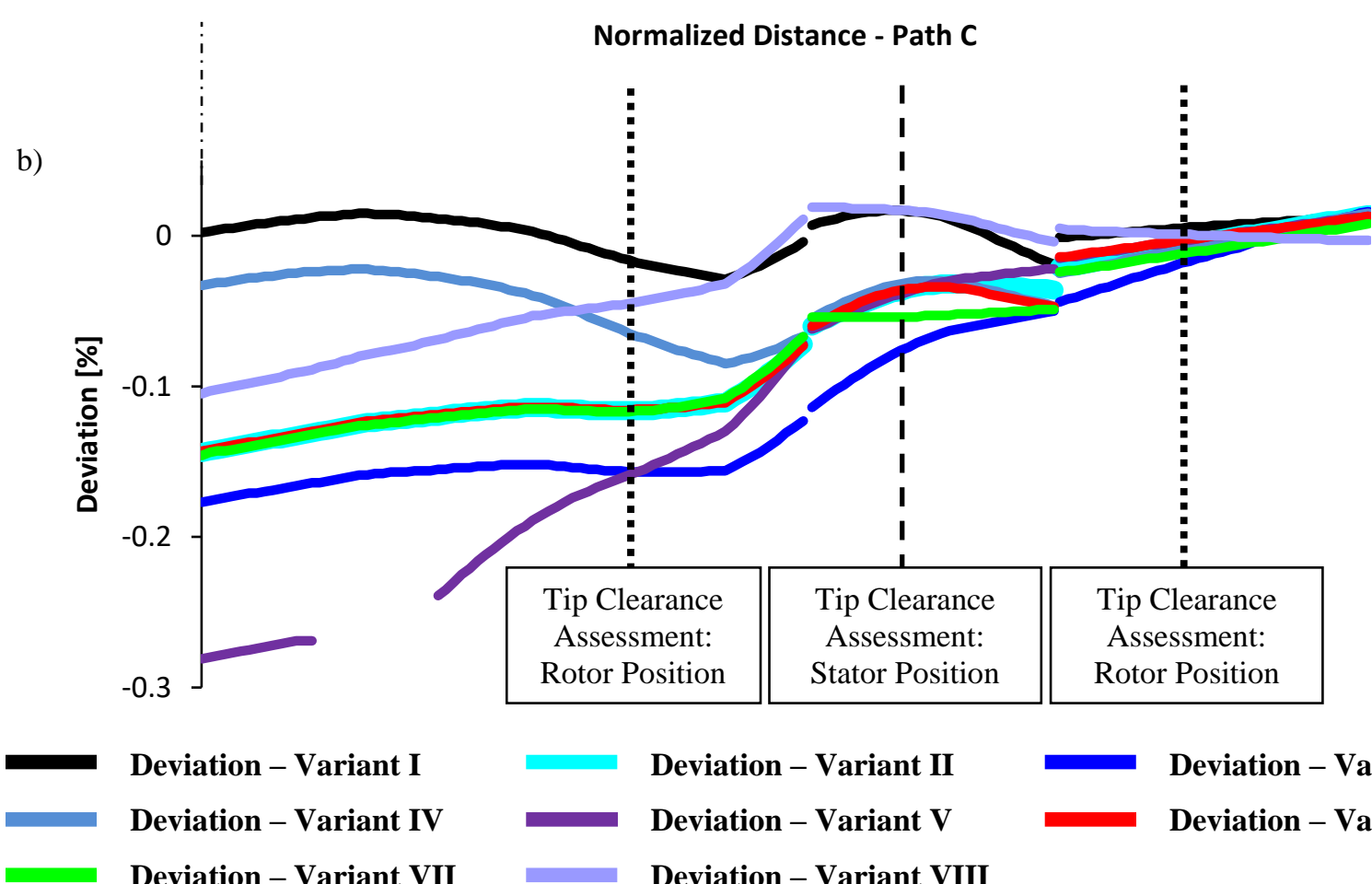
Deviation - Variant I
Deviation - Variant IV
Deviation - Variant VII

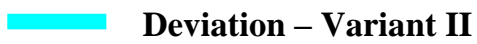

Deviation - Variant III

Deviation - Variant V

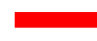

Deviation - Variant VI

Deviation - Variant VIII

Fig. 12: a) Application of Model Simplifications for Transition from 3D to 2D Axisymmetric Finite Element Modeling; b) Development of Deviation in Radial Displacement with Transition from 3D to 2D Axisymmetric Finite Element Modeling. 


$$
\text { Deviation }=\frac{\text { Radial Displacement }_{3 D \text { Model }}-\text { Radial Displacement }_{2 D \text { Model }}}{\text { Radial Displacement }}
$$

The 2D axisymmetric Finite Element model matches the detailed 3D models very well with an increased approximation towards the end of path $\mathrm{C}$. The effects of bolted joints and bleed air ports on radial casing displacement correlate, and varying the CQ of the stated 3D features has the most impact on radial casing displacement when inspecting the curves of Variant III and V in Figure 12b. In total, the peak value of deviation in radial displacement with a magnitude of $0.28 \%$ caused by the covered 3D features is considered to be negligible.

When taking the reduced numerical effort after transitioning from 3D to 2D axisymmetric Finite Element modeling into account, a reduction of at least $99.9 \%$ in total CPU time consumption was assessed in favor of the $2 \mathrm{D}$ model variant. Regarding comparability, attention was paid to solving computational grids with equivalent cross-sectional mesh resolution. Less nodal quantity even at the computationally very expensive contact surfaces is the decisive reason for this observation.

\section{Conclusion and Prospects}

Proceeding from the 3D model of a generic axial compressor casing, the 3D feature effects of bolted joints and bleed air ports on radial displacement during steady state engine operation were investigated via Finite Element Method. Parameter studies varying the CQ and $\varnothing$ of the specified 3D features were conducted for quantification of the impact on radial displacement. Furthermore, parameter dependent tendencies of casing expansion behavior were identified, and approaches for model simplification from 3D to 2D axisymmetric Finite Element modeling were applied.

Evaluating the circumferential tracks of the 3D features concentrated in this work, bolted joints generally induce a local peak in radial displacement while bleed air ports show exactly the opposite behavior. A growth of bolted joint $\emptyset$ causes a rise in radial displacement. Once the casing geometry is equipped with bleed air ports, an increase in radial displacement is ascertained as a consequence of enlarged bleed air port $\varnothing$. The fact that increasing the CQ leads to a reduction in radial displacement is a finding that both 3D features share. Detailed analysis ascribed the dominant reason for this reaction of CQ variation to a cooling effect that occurs due to an increased surface wetted with bleed air. Moreover, a correlation between bleed air ports and bolted joints is to assess and can be traced back even in longitudinal direction. Concerning radial displacement manipulation, the 3D feature effect of bleed air ports is more intense than the equivalent of bolted joints, and the impact of CQ noticeably exceeds the consequence of $\varnothing$ variations. Altogether, the investigated effects of bleed air ports and bolted joints on radial casing displacement range in the magnitude of manufacturing tolerances. Matching the calculated 3D model variants with a simplified 2D axisymmetric Finite Element model results in a good approximation worth to resume towards the project intention of prompt tip clearance assessments in the design phase of high-pressure compressor casings.

Future investigations at the Institute will address the effect of further 3D features on radial casing displacement and corresponding methods for model simplification. Currently, essential parameter studies will be continued which cover the

effect of varied boundary conditions at the stator model and cavity. Split casings with variable stator vanes are intended to be elaborated in detail next.

\section{References}

[1] S. Weihard, Bauweisenkatalog, Internal Report, Institute for Turbomachinery and Flight Propulsion, Technische Universität München, Germany, 2013.

[2] Department of Defense, Military Handbook-Metallic Materials and Elements for Aerospace Vehicle Structures: MIL-HDBK-5H. USA, 1998.

[3] J. R. Davis, Nickel, Cobalt, and Their Alloys. ASM international, 2000.

[4] Special Metals, Inconel® alloy (2019, April 12). [Online]. Available: www.specialmetals.com/assets/smc/ documents/alloys/inconel/inconel-alloy-718.pdf 